\title{
Management of a patient with Sheehan's syndrome and diabetes insipidus complicated by recurrent hyponatremia
}

\author{
Butheinah A Al-Sharafi(D, Faiza Askar and Ahmed A Qais \\ 'Department of Internal Medicine, School of Medicine and Health Sciences, Sana'a University, Sana'a, Yemen
}

Correspondence should be addressed

to B A Al-Sharafi

Email

balsharafi@hotmail.com

\begin{abstract}
Summary
A 38-year-old female was initially seen in the intensive care unit after severe postpartum hemorrhage. She was initially diagnosed to have Sheehan's syndrome and after discharge, she was diagnosed to have a vesicovaginal fistula which initially caused a delay in diagnosing diabetes insipidus in the patient because she was having urinary incontinence. The patient had the vesicovaginal fistula repaired and was on replacement with levothyroxine, prednisone, and desmopressin. Years after her diagnosis, the patient experienced recurrent episodes of hyponatremia in the setting of desmopressin therapy. This case highlights the challenges of diagnosing diabetes insipidus in a patient with Sheehan's syndrome and a vesicovaginal fistula, as well as the long-term management of central diabetes insipidus in a resource-limited setting.
\end{abstract}

\section{Learning points:}

- Sheehan's syndrome is rarely associated with diabetes insipidus, and in our patient, it was initially missed due to a vesicovaginal fistula which caused urinary incontinence.

- Water intoxication is more common in young children and older adults but can occur years after initiating treatment with desmopressin in adults and should be kept in mind when treating patients with hyponatremia who have hypopituitarism associated with diabetes insipidus.

- Water intoxication is much more common in patients with diabetes insipidus being treated with intranasal desmopressin than in those using the oral preparations.

\section{Background}

Sheehan's syndrome refers to postpartum hypopituitarism which occurs as a result of ischemic pituitary necrosis due to severe postpartum hemorrhage $(1,2)$. It is still commonly seen in underdeveloped and developing countries (3). It is characterized by varying degrees of anterior pituitary dysfunction and rarely associated with diabetes insipidus (3) which is estimated to occur in $5 \%$ of the cases $(3,4)$. Hyponatremia in patients with hypopituitarism can occur due to hypothyroidism or hypoadrenalism (5). In addition, water intoxication can be a cause of hyponatremia in patients with diabetes insipidus being treated with desmopressin especially the intranasal form (6). To the best of our knowledge, we are reporting the first case to have Sheehan's syndrome with diabetes insipidus and also a vesicovaginal fistula. Also, our patient developed recurrent water intoxication causing hyponatremia which occurred years after her initial diagnosis.

\section{Case presentation}

A 38-year-old female was first seen in 2009 in the intensive care unit in the University of Science and Technology 
Hospital in Yemen. An endocrinology consult was requested on a patient who had an obstructed labor which required a Cesarean section and was followed by severe postpartum hemorrhage and a hysterectomy. The patient had been resuscitated with blood and i.v. fluids but continued to have hypotension. The doctor in the intensive care unit suspected that the patient may have Sheehan's syndrome and started the patient on steroids which caused the patient to stabilize, but no workup had been done to confirm the diagnosis prior to starting the hydrocortisone.

\section{Investigation}

Initial blood work after starting hydrocortisone (Table 1) showed a low LH and FSH with a normal cortisol and normal TSH and FT4. The patient improved and was discharged on prednisone $20 \mathrm{mg} /$ day and was advised to follow-up 1 week later.

The patient was not seen till 2 weeks later complaining of fatigue and was found to have a low TSH, a low FT4, and very low estradiol (Table 1) and was started on thyroxine of $75 \mu \mathrm{g}$ daily in addition to tapering the prednisone slowly to reach $5 \mathrm{mg}$ daily. Growth hormone level was not measured since replacement is not usually given to adults in Yemen. All electrolytes were checked including calcium and sodium levels and were all within the normal range.

The patient called the clinic a few days later complaining of polyuria and polydipsia. She reported blood glucose

Table 1 Initial blood work of patient and follow-up blood work after 2 weeks.

\begin{tabular}{|c|c|c|c|}
\hline Hormones & Normal levels & Baseline* & Follow-up ${ }^{\dagger}$ \\
\hline TSH, mU/L & $0.5-5.0$ & 2.04 & 0.125 \\
\hline Free T4, ng/dL & $0.7-1.9$ & 1.09 & 0.658 \\
\hline Cortisol, $\mu \mathrm{g} / \mathrm{dL}$ & $5-25$ & $5.34^{\ddagger}$ & $0.1^{\ddagger}$ \\
\hline Prolactin, ng/mL & $<25$ & 4.13 & 2.28 \\
\hline LH, IU/L & $5-25$ & $<0.1$ & 0.502 \\
\hline FSH, IU/L & $4-25$ & 0.43 & 0.378 \\
\hline Estradiol, pg/mL & $15-350$ & 59.5 & 0.2 \\
\hline IGF-1 & & ND & \\
\hline Growth hormone & & ND & \\
\hline Sodium, mEq/L & $135-145$ & 137 & \\
\hline Calcium, mg/dL & 8.6-10.3 & 9.2 & \\
\hline $\begin{array}{l}\text { Fasting blood } \\
\text { glucose, mg/dL }\end{array}$ & $70-99$ & 90 & \\
\hline Serum osmolality & & ND & \\
\hline Urine osmolality & & ND & \\
\hline
\end{tabular}

*Measured on November 17, 2009; 'Measured on December 5, 2009; ₹On steroids.

ND, not determined. levels that had been checked, $95 \mathrm{mg} / \mathrm{dL}$ fasting and $125 \mathrm{mg} /$ $\mathrm{dL}$ post-prandially. On further questioning, it seemed that the patient was incontinent to urine and had continuous dribbling of urine. The patient was suspected to have a vesicovaginal fistula and was referred to a gynecologist who confirmed the diagnosis.

The patient was not seen for a month and she came back still complaining of increased thirst and drinking very large amounts of fluid each day and passing large amounts of urine. The patient was suspected to have diabetes insipidus, and we discussed with the patient doing an overnight water fasting test to check the serum/urine osmolality and sodium levels since the water deprivation test was not available in Yemen, but she stated that she could not spend more than an hour without drinking so we decided to give an intranasal desmopressin challenge (1 puff $10 \mu \mathrm{g}$ once daily at bedtime which was increased to twice daily a couple of days later) which caused a marked improvement in her condition with decreased thirst and a decrease in the amount of urine. The patient at that time traveled abroad for the repair of the vesicovaginal fistula which was successful and was switched to hydrocortisone and she continued on desmopressin nasal spray (10 $\mu \mathrm{g}$ bid) and levothyroxine $75 \mu$ g daily. Magnetic imaging of the pituitary was done 2 weeks after the diagnosis and was reported as normal with no comment on the posterior pituitary bright spot.

\section{Treatment}

The patient was followed up over the following years with some adjustment of the levothyroxine dose and hydrocortisone was switched to prednisone $5 \mathrm{mg}$ daily because it was not always available and the patient was advised regarding stress dosing of the glucocorticoids during acute illnesses, trauma, or surgery. In the early years of her disease, she was taking desmopressin only once daily at times due to the expense of the drug and she sometimes felt that one puff $(10 \mu \mathrm{g})$ daily would be enough at night but she soon started to use it twice daily due to increased polyuria and polydipsia. Also, at times, she would switch to oral desmopressin $(200 \mu \mathrm{g}$ twice daily) according to the availability in the market, but for the last 3 years, she had been taking intranasal desmopressin which she would buy from abroad. The patient did not want to take any estrogen hormone replacement therapy but was put on vitamin $\mathrm{D}$ and calcium replacement. 


\section{Outcome and follow-up}

The patient continued to follow up every 6 months requiring some adjustment in the levothyroxine dose and requiring stress doses of prednisone if she was ill she also continued on desmopressin bid (intranasally for the last 3 years). In 2020, she called the clinic saying she was having severe headaches and fatigue. Her free T4 level was normal ranging $1.62 \mathrm{ng} / \mathrm{dL}$. MRI scan of the brain and pituitary was reported as normal. The sodium level was done and was low at $120 \mathrm{mEq} / \mathrm{L}$. It was initially thought that she was having hypoadrenalism or water intoxication. The patient had severe fatigue, but other symptoms of hypoadrenalism were not present such as hypotension and nausea and she did not show any improvement in her symptoms on increasing her dose of prednisone and giving her hydrocortisone intravenously. Water intoxication from the desmopressin was considered, and on further questioning, the patient stated that she took her evening dose regularly (1 puff of desmopressin $10 \mu \mathrm{g}$ ), but in the morning, she would delay her dose until she became symptomatic and was having polydipsia and polyuria then she would take dose usually $16 \mathrm{~h}$ after her evening dose. The patient was advised to take desmopressin every $12 \mathrm{~h}$ and not to wait till she was symptomatic (on questioning it seemed that she continued to drink large amounts of fluid for a while even after she took her dose of desmopressin and we thought that this may be a factor in causing the water intoxication) and she was also advised to reduce her fluid intake to $800 \mathrm{~mL}$ daily. The sodium level was repeated a few days later the levels had improved to 133 $\mathrm{mEq} / \mathrm{L}$ and the patient felt much better. The patient did well for a few months when she started to complain of fatigue again and the sodium level had decreased to $127 \mathrm{mEq} / \mathrm{L}$, the patient stated that she was taking desmopressin regularly most of the time. She improved again on decreasing fluid intake and a week later, her sodium level increased to $134 \mathrm{mEq} / \mathrm{L}$; the patient was also advised to drink fluids only if thirsty and has been asymptomatic for the last 4 months. We considered using a lower dose using a rhinyl tube (dose range 1-10 $\mu \mathrm{g}$ ) or a metered-dose spray (2.5-10 $\mu$ g per spray) but these were not available in Yemen. We have considered switching the patient to oral desmopressin during the daytime if she continues to have recurrent attacks of hyponatremia since the risk of hyponatremia is increased with the nasal formulation because of higher variability in absorption from the nasal mucosa.

\section{Discussion}

Sheehan's syndrome is rarely seen in developed countries due to improved obstetric care (7) but is still seen commonly in Yemen, and most of the cases present to us many years after having postpartum hemorrhage. Our case was also associated with a vesicovaginal fistula, another complication of obstructed labor that has not been reported previously in a patient with Sheehan's syndrome. The patient being incontinent to urine caused a delay in diagnosing the associated diabetes insipidus which occurs rarely in Sheehan's syndrome. The patient later developed water intoxication which was initially misdiagnosed as hypoadrenalism.

Most cases of water intoxication occur in children or older-aged patients. In children, it has been found that most cases occur when using intranasal or i.v. desmopressin, none of the patients had received oral desmopressin (8). We did not check sodium levels in our patient regularly and previous episodes of fatigue that the patient had may have been water intoxication that had been missed or misdiagnosed as hypoadrenalism. It should be kept in mind that water intoxication may also occur in adults and may be quite common as was shown in a retrospective study on 70 adult patients with central diabetes insipidus in which 32 of the patients developed hyponatremia with 10 of them having severe hyponatremia (sodium $<125$ $\mathrm{mEq} / \mathrm{L}$ ) and this was more common in females. This study also showed that $84 \%$ of the patients who developed hyponatremia were found to be on the nasal spray or nasal drops with only 16\% taking oral desmopressin (9). Our patient was on intra-nasal desmopressin at the time she developed the hyponatremia and in the past, she had been on oral desmopressin. The bioavailability of the intranasal formulation $(6.00 \pm 2.29 \%)$ is higher than the oral preparation $(0.16 \pm 0.17 \%)$. Oral administration of desmopressin in doses 10-20 times the intranasal dose provides adequate blood levels of desmopressin to control polyuria in central diabetes insipidus (6). Due to the ease of administration of the oral formulations, they are the preferred route for the treatment of most patients (6). The risk of hyponatremia can be reduced by adhering to the indications, dosing recommendations, and precautions when prescribing desmopressin. Starting at the lowest dose and increasing only if required with caution (10). Patients should be advised on the warning signs of hyponatremia (headache, nausea, weight gain, and vomiting), also they should be warned against overdosing especially when using the intranasal formulation (10). The intranasal formulation 
is associated with large variability in absorption which can cause an unwanted increased duration of action, which can result in hyponatremia which is less likely to occur in patients taking the oral formulation $(10,11)$. In one study on patients with central diabetes insipidus taking the oral formulation of desmopressin mild hyponatremia occurred in $27 \%$ of the patients with intact thirst and $14.6 \%$ developed more significant hyponatremia but only $5.8 \%$ were admitted due to complications related to hyponatremia, the authors of the study recommended withholding one dose of desmopressin a week to allow diuresis (12). We have currently advised our patient to do the same.

In patients with hypopituitarism associated with diabetes insipidus who develop hyponatremia it should be kept in mind that water intoxication might be a cause even in adults with intact thirst.

\section{Declaration of interest}

The authors declare that there is no conflict of interest that could be perceived as prejudicing the impartiality of the research reported.

\section{Funding}

This case report did not receive any specific grant from any funding agency in the public, commercial or not-for-profit sector.

\section{Patient consent}

Written consent has been received from the patient.

\section{Author contribution statement}

$B$ A and F A treated and followed-up the patient, B A wrote the script with the assistance of $\mathrm{FA}, \mathrm{A} Q \mathrm{Q}$ reviewed the literature and also helped in the care of the patient. All the authors critically reviewed and approved the final manuscript.

\section{References}

1 Karaca Z, Laway BA, Dokmetas HS, Atmaca J \& Kelestimur F. Sheehan syndrome. Primer 20162 1. (https://doi.org/10.1038/nrdp.2016.92)

2 Robalo R, Pedroso C, Agapito A \& Borges A. Acute Sheehan's syndrome presenting central diabetes insipidus. BMJ Case Reports 2012. (https:// doi.org/10.1136/bcr-2012-007022)

3 Atmaca H, Tanriverdi F, Gokce C, Unluhizafci K \& Kelestimur F. Posterior pituitary function in Sheehan's syndrome. European Journal of Endocrinology 2007156 563-567. (https://doi.org/10.1530/EJE-060727)

4 Kelestimur F. Sheehan's syndrome. Pituitary 20036 181-188. (https:// doi.org/10.1023/b:pitu.0000023425.20854.8e)

5 TZvi-Ran IR, Olchowski J, Fraenkel M, Bashiri A \& Barski L. A rare cause of postpartum acute hyponatremia. EDM Case Reports 20192019 18-0124. (https://doi.org/10.1530/EDM-18-0124)

6 Oiso Y, Robertson GL, Norgarrd JP \& Juul KV. Clinical review: treatment of neurohypophyseal diabetes insipidus. Journal of Clinical Endocrinology and Metabolism 201398 3958-3967. (https://doi. org/10.1210/jc.2013-2326)

7 Kumar S, Burrows D, Dang S \& Simmons D. Sheehan syndrome presenting as central diabetes insipidus: a rare presentation of an uncommon disorder. Endocrine Practice 2011 17 108-114. (https://doi. org/10.4158/EP10015.CR)

8 Thumfart J, Roehr CC, Kapelari K, Querfild U, Eggert P \& Muller D. Desmopressin associated symptomatic hyponatremic hypervolemia in children: are there predictive factors? Journal of Urology 2005174 294-298. (https://doi.org/10.1097/01.ju.0000161213.54024.7f)

9 Masri-Iragi H, Hirsch D, Herzberg D, Lifshitz A, Tsvetov G, Benbassat C\& Ilan Shimon I. Central diabetes insipidus: clinical characteristics and long-term course in a large cohort of adults. Endocrine Practice 201723 600-604. (https://doi.org/10.4158/ EP161555.OR)

10 Walle JV, Stockner M, Raes A \& Norgaard JP. Desmopressin 30 years in clinical use: a safety review. Current Drug Safety 20072 232-238. (https://doi.org/10.2174/157488607781668891)

11 Kataoka Y, Nishida S, Hirakawa A, Oiso Y \& Arima H. Comparison of incidence of hyponatremia between intranasal and oral desmopressin in patients with central diabetes insipidus. Endocrine Journal 201562 195-200. (https://doi.org/10.1507/endocrj.EJ14-0368)

12 Behan LA, Sherlock M, Moyles P, Renshaw O, Thompson CJT, Orr C, Holte K, Salehmohamed MR, Glynn N, Tormey W, et al. Abnomal plasma sodium concentrations in patients treated with desmopressin for cranial diabetes insipidus: results of a long-term retrospective study. European Journal of Endocrinology 2015172 243-250. (https:// doi.org/10.1530/EJE-14-0719)

Received in final form 30 July 2021

Accepted 17 August 2021 